\title{
Mediating Tragedy: Facebook, Aboriginal Peoples and Suicide
}

\author{
Bronwyn L Carson \\ University of Wollongong \\ bcarlson@uow.edu.au
}

Terri Farrelly

The Echidna Group: Indigenous Research and Development Consultancy drterrifarrelly@gmail.com

\author{
Ryan Frazer \\ University of Wollongong \\ rfrazer@uow.edu.au
}

\author{
Fiona Borthwick \\ University of Wollongong \\ fiona@uow.edu.au
}

\section{Abstract}

Some Australian Aboriginal communities experience suicide rates that are among the highest in the world. They are also, however, avid social media users - approximately 20 percent higher than the national average. This article presents some preliminary findings from a current national study, funded by the Australian Research Council, titled Aboriginal identity and community online: a sociological exploration of Aboriginal peoples' use of online social media. The purpose of the study is to gain insights into how Aboriginal people utilise and interact on social media, and how these technologies can assist with suicide prevention strategies. It found that Aboriginal people are engaging with Facebook to both seek and offer help for issues relating to suicide and self-harm. An existing continuum of suicide prevention strategies was evident-from light emotional support to direct suicide intervention involving health services. These strategies can be leveraged to implement effective and appropriate suicide prevention programs.

Keywords: social media; Indigenous; Aboriginal; social networking; suicide

\section{Introduction}

In this article we present some preliminary findings from a current national study, funded by the Australian Research Council, titled Aboriginal identity and community online: a sociological exploration of Aboriginal peoples' use of online social media. The purpose of the study is to gain insights into the ways in which Australian Aboriginal people utilise and interact on social media. Results demonstrate that Aboriginal people are avid social media users, that they use social media for a range of activities, including those associated with mental health and well-being (Carlson 2014; Carlson and Frazer 2015). Use of social media is clearly not a peculiarity among some Aboriginal people but rather a daily activity for the vast majority, with Facebook being the most popular site (Carlson 2013).

While there is a growing body of research examining suicide and social media (see for example, Robinson et al. 2014), there is a paucity of studies that focus on links between Aboriginal people's use of social media and suicide. Given the high rates of both suicide and social media use in Aboriginal communities (The Elders Report 2014:7), this is surprising. Our research findings revealed that Aboriginal people are engaging with Facebook to both seek and offer help for issues relating to suicide and self-harm. An existing continuum of suicide prevention strategies was evident-from light emotional support to direct suicide intervention involving health and emergency services. The results thus demonstrate that online social technologies, such as Facebook, offer Aboriginal users new pathways to engage in help-seeking and help- 
giving behaviours. We propose these online practices suggest social media presents a potentially efficacious platform for developing culturally appropriate suicide interventions and prevention programs.

\subsection{Background}

\subsubsection{Aboriginal Suicide}

Until the 1970s suicide among Aboriginal people was a relatively rare phenomenon (ElliottFarrelly 2004). Now, however, some Aboriginal communities experience rates of suicide and self-harm that are up to 40 percent higher than the general Australian population (Carlson 2014), making them among the highest in the world (The Elders Report 2014:7). Reducing suicide and suicidal behaviour among Aboriginal people is now a public health priority for all Australian governments.

\subsubsection{Differing Epidemiologies, Methods, Risk Factors and Aetiological Theories}

Key research conducted into Aboriginal suicide and self-harm behaviour in northern Queensland (Hunter et al. 2001) and New South Wales (Tatz 2001) uncovered myriad factors which differentiate Aboriginal suicide from non-Aboriginal suicide, in terms of epidemiology, methods, risk factors and aetiological theories. These factors impact the (in)effectiveness of current prevention measures, the designs of which have largely been based on western cultural understandings of and attitudes towards suicide and self-harm behaviour and prevention.

Many of the mainstream social risk factors for suicide do not appear to be relevant to Aboriginal people and their communities (Tatz 2001). As Tatz (2001) demonstrates, there exists a separate Aboriginal suicidology-perhaps even suicidologies-which is further complicated by significant cultural and socio-economic variation between regions and communities. The sudden onset and increase in Aboriginal suicide rates since the 1970s is often blamed on the rapid social transformation enforced upon Australia's Indigenous peoples, exacerbated by social and economic disadvantage, systemic oppression and racism (Hunter et al. 2001; Tatz 2001; Pink and Allbon 2008). However, there have been few careful and systematic research studies into the causes of Aboriginal suicide.

It has long been held that psychiatric illness and other pathologies are present in cases of attempted and completed suicide. However, research has shown that while this may be the case in the general population, it does not appear to be applicable in Aboriginal communities (Tatz 2001). Furthermore, while depression is often cited as the precipitating cause of Aboriginal suicidality, there has been little reference to the cultural context and meanings of depression-like behaviour (Reser 1991). Thus Tatz (2001) argues that Aboriginal suicidality is not always the domain of mental health. He suggests there is little evidence of clinical depression in cases of Aboriginal suicide, just as there appears to be little or no correlation between Aboriginal suicide and diagnosable mental illness more broadly. Rather, Aboriginal suicide is often impulsive and frequently occurs in the context or aftermath of intoxication (Hunter et al. 2001; Tatz 2001).

This research points to the need to understand Aboriginal suicide in its cultural context, rather than uncritically imposing western notions of health and well-being. An emerging literature is providing a strong foundation for understanding Aboriginal and Torres Strait Islander perspectives of well-being (Dudgeon and Kelly 2014; Ranzijn, McConnochie and Nolan 2009; Westerman 2010). Increasing consideration is being paid to how Aboriginal people's diverse notions of well-being dynamically interact with their ways of knowing, doing, and being (Martin 2008; Moreton-Robinson and Walter 2008); some research has paid particular attention to the role of information and technology (Radoll 2012; Rigney 2011). Thus there is increasing academic acknowledgement of the need to establish a more robust knowledge of the diverse understandings of health Aboriginal people draw on in their help-seeking and helpgiving behaviours. 


\subsubsection{Aboriginal People and Social Media}

Research tells us Aboriginal people have always been early adopters of technology, with social media being no exception (Pascoe 2014). In 2010 the Australian Institute of Aboriginal and Torres Strait Islander Studies (AIATSIS) hosted a conference on Information Technologies and Indigenous Communities' (J uly 13th to 15th 2010). Among the topics discussed, the 'Facebook and Social Networking' theme claimed that sites such as Facebook:

... are going to be a critical part of life in your community in the future. New technology allows the creation of new linkages that have not been seen in our cultures before (2010:30).

Across Australia, Aboriginal and Torres Strait Islander use of social media is approximately 20 percent higher than the national average and in remote communities over 60 percent of the population are active Facebook users (Callinan 2014). Social media is transforming the way we communicate with each other and the way in which we are connected to other people at a local, regional, national and global level. Certainly for Aboriginal and Torres Strait Islander people, Facebook and other social media provide a site of connection to others and allow users to maintain contact and culture across both vast distances and time, increasing social connectivity (Carlson 2013).

The rapid advances in mobile technologies, and the uptake of these by Aboriginal youth in particular, can be evidenced in many Aboriginal communities (see Kral 2011). While this is not to suggest there is no 'digital divide', it does counter any assumptions that Aboriginal people may have little interest in the possibilities of technology and the online environment. Kral's (2011:5) research into how Aboriginal youth in remote Australia utilise digital technologies and new media documented popular use of Facebook as a platform to:

...upload their multimedia productions, comment on each others' mobile phone 'pics' and announce the immediacy of their activities with online chat...[T]hey are also using these channels to air their thoughts and the cultural activities and concerns of their community (Kral 2011:5).

Carlson (2013:147) likewise observed that the use of social media by Aboriginal people has become an "everyday, typical activity". Aboriginal people are also participating in political and cultural activities on social media-particularly Facebook (see also Carlson 2013, 2014; Carlson and Frazer 2015). In a recent publication examining Aboriginal people, social media and practices associated with death, Carlson and Frazer (2015) found that Aboriginal people were using social media for notifications of deaths and funerals, offering condolences and extending support, and grieving and healing. They also revealed that social media can be both a productiveand problematic space for Aboriginal people. While social media provided an online space where families and extended kin could assemble, it was also a contested space where competing cultural understandings, values and performances of respect and bereavement played out publicly.

Social media can also provide a space where Aboriginal people become subject to abuse. Carlson (2014) has raised the issue of 'R.I.P Trolls' (i.e., when instigators post abusive comments and memes on memorial pages dedicated to the deceased), racism and abuse targeted towards Aboriginal people on social media. Rauch and Schanz (2013) argue that it is not difficult to locate negative comments about minority groups on social media, noting that at times posts can be subtly negative but are often overtly racist. Australia's Race Discrimination Commissioner has also raised the issue of cyber racism, stating that there has been an increase in complaints about online racism (Emery 2014). It is in this context that Georgatos (2014) powerfully argues that racism is implicated in some suicides in Aboriginal communities.

\subsubsection{Aboriginal Help-Seeking and Social Media}

Help-seeking behaviour refers to the coping mechanisms an individual displays in the face of distress or pressure, or the communicative process of seeking help from others in the event of 
particular difficulty or emotional distress (Gourash 1978). Globally, research has found that many people feel empowered while help-seeking online and are more confident in accessing information and talking about sensitive topics (Gould et al. 2002). There is emerging evidence that technology-based interventions, such as offering information or access to professional advice, could be effective (Griffiths et al. 2010).

In an investigation into Aboriginal suicide and self-harm, Farrelly (2008) categorised helpseeking as either formal or informal. Formal help-seeking involves services and professionals who have been formally trained as help sources, such as counsellors, psychologists, general practitioners and the like. Informal help-seeking, on the other hand, involves untrained help sources such as family, friends or other community members and social networks.

Several studies have outlined the barriers that may hinder Aboriginal and Torres Strait Islander people from engaging in formal, face-to-face help-seeking (Zubrick et al. 2005; Rickwood et al. 2007; Lumby and Farrelly 2008 \& 2009; Farrelly 2008). Mistrust of government services and intergenerational trauma related to past policies of removal and racism can impact help-seeking behaviours (Zubrick et al. 2005). Intergenerational stigma and feelings of shame associated with help-seeking remains a barrier in all age groups, but particularly among Indigenous youth (Price and Dalgleish 2013). Research has thus indicated that Aboriginal and Torres Strait Islander help-seeking is typically more informal than that practiced by non-Indigenous people (Farrelly 2008; Rickwood et al. 2007). This is largely due to a general lack of help-seeking skills and a reluctance to invite the scrutiny of mainstream service providers into homes, lives and communities (Lumby and Farrelly 2008). The onus of help-giving then, typically falls to family, friends and social networks. Finding solutions to the barriers that impede Aboriginal and Torres Strait Islander help-seeking is crucial given the high level of unmet need (Rickwood et al. 2007: 35). We suggest social media may offer some such solutions.

While researchers have recently begun to examine the potential of social media for Aboriginal and Torres Strait Islander help-seeking, the area remains underexplored, with little research involving large cohorts of Aboriginal and Torres Strait Islander social media users. Studies have largely focussed on the help-seeking behaviours of Aboriginal and Torres Strait Islander youth specifically. Price and Dalgleish's (2012) research, for instance, revealed that Indigenous youth actively accessed self-help information online and peer support opportunities through social networking. Edmonds et al's (2012) study of Aboriginal youth's use of mobile devices and social networking found that Aboriginal youth used social media for "maintaining connections and for pathways to assist them when facing big decisions" (2012:12) and that social media offered a sense of community that could provide support in stressful situations. Young Aboriginal people were using their online networks in what could be described as informal help-seeking.

While this research is invaluable, little is known about the specifics of the complex relationships between Aboriginal and Torres Strait Islander use of social media and helpseeking and help-giving practices. It is also not known whether the same offline barriers that nurture a reluctance to seek formal help sources also exist online on social media. Nor is it known whether informal help sources on social media are providing appropriate advice and/ or intervention. Additionally, little is known about the role of the informal help sources and helpgiving on social media and whether social media provides a forum where the needs of the helpseeker can be met.

This knowledge gap is not isolated to Australia. Internationally, little is known about Indigenous help-seeking behaviours on social media. Research on Indigenous peoples and social media is an emerging field and generally focuses on investigating the impact that social media is having on cultural practices (see, O'Carroll 2013) or using technology to reach Indigenous people and communities for health related issues (see, Rushing and Stephens 2011). Others map Indigenous use of social media for communicating, socialising and participating in cultural practices, when vast distances isolate communities (see, Molyneaux et al. 2014). What we can ascertain from these studies is that Indigenous groups are active social 
media users and their online engagements include connecting with each other socially and culturally - and is likely to include practices of help-seeking and help-giving.

\subsubsection{Suicide and Social Media}

Facebook, like other social media sites, is a platform where users can update their networks about how they are feeling by regularly posting 'status updates'. Manago et al. (2012:369) found "emotional disclosure", or "self disclosure", to be the major function of Facebook's status update feature. In a recent UK study it was revealed that social media may feed anxiety and increase feelings of inadequacy (Paddock 2012). The researchers found that over half of the respondents claimed social media made them feel less confident and that they spent time comparing their lives to their online networks. Further, the research also found that after online confrontations the participants reported experiencing difficulties in personal relationships (Paddock 2012). Conversely, Professor Jessica Vitak of the College of Information Studies at the University of Maryland argues that the relationship between social media and mental health is far too complex to suggest that it has blanket effects across users. She has found that, for many people, social media can provide social benefits in terms of social and emotional support and maintaining relationships that would have faded away without the technology. She also concedes, however, that in some instances, such as those involving cyber bullying and online harassment, the effects on well-being may be negative.

In a comprehensive review of the literature associated with social media and suicide, it was revealed that some people use social media for communicating suicidal ideations. This study also reported a correlation between cyber bullying and suicide ideation (Robinson et al. 2012:12), noting the potential for harm must be taken seriously (2012:18). There are several documented cases of people posting status updates that are intended to act as suicide announcements or suicide notes. A recent news article in The Australian reported Facebook being used by Aboriginal people to post messages of suicidal intent, with one young girl posting, "Feel lost right now hang myself" (Martin 2014). Such announcements are generally visible to large numbers of other users within that person's network-from dozens to thousands of others. As Ruder et al. (2011) point out, this offers a potential opportunity for people to intervene in the realisation of the suicide risk, either through directly contacting or approaching the person, or involving emergency services. Similarly, Robinson et al. (2014:7) found that social media platforms have the potential to enable users to "access information, support and counselling and to share their experiences in a flexible, timely and readily accessible format". Robinson et al's (2014:7) studyalso found that help seeking and responding was a "valuable and therapeutic feature" of social media.

In the UK, several public cases of suicide announcements on Facebook prompted UK charity Samaritans to team up with Facebook to provide help for those feared to be in danger (see, Luxton et al. 2012). From March 2011, UK users were able to report concerns prompted by their friends' posts through the Facebook Help Centre. Facebook would then put Samaritan counsellors in touch with those in distress. A comparable initiative is currently being tested in the United States. A collaboration between Facebook and researchers from the School of Social Work at the University of Washington has seen the development of suicide prevention and support tools (Worrall 2015). If a user identifies a post that causes them concern over another's safety, they can then report it to Facebook for review. If the post is found to indicate distress or suicidal thoughts, Facebook sends the person a series of notifications offering advice (Worrall 2015). Although the effectiveness of these or similar programs has not yet been assessed, these efforts to leverage social media's power for improving the well-being of its users could be promising.

While these social media intervention initiatives appear promising, there is also need for caution when dealing with vulnerable groups, such as Aboriginal and Torres Strait Islander people. Luxton et al. (2012:197) note that social media may pose a risk to vulnerable groups and argue that more research is needed focussing on both social media's "negative and positive influences". They suggest that examination of "sub-groups that might be most vulnerable to suicide promoting influences of social media is also warranted" (Luxton et al. 2012:197). 


\section{Methodology \& Research Design}

To explore Aboriginal people's use of social media for help-seeking and help-giving, this study utilises Nakata's (2007) notions of an Indigenous Standpoint Theory and the Cultural Interface as both an entry point for inquiry and a tool for analysis. Nakata's conceptualisation of a Standpoint Theory for Indigenous research and researchers provides a way for understanding the contemporary complexities of Indigenous experience and struggles. Indigenous Standpoint Theory is a method for Indigenous researchers to critically assess the corpus of knowledge written about us. Nakata suggests, Indigenous Standpoint Theory can assist a way to theorise

knowledge from a particular and interested position-not to produce the 'truth' of Indigenous position but to better reveal the workings of knowledge and how understanding of Indigenous people is caught up and implicated in its work (2007:215).

Indigenous Standpoint Theory is complemented by and perhaps best understood alongside his concept of the Cultural Interface, a term he coined to denote the everyday site of struggle that continues to confine colonised people. The Cultural Interface is located at

the intersection of the Western and Indigenous domain...the place where we live and learn, the place that conditions our lives, the place that shapes our futures and more to the point the place where we are active agents in our own lives-where we make decisions-our lifeworlds (Nakata 2002:285).

To explore the diversity of everyday experiences of Aboriginal social media users, data was collected through a variety of qualitative methods. Following formal ethics approval from the University of Wollongong's ethics committee, participants were recruited through contacting key figures from each community, who were thereafter employed as one-off research assistants. This method was deemed both ethical and effective. Respected persons in communities could communicate the purposes and content of the research project to potential participants and help interviewers conduct the interviews in an appropriate manner. In this way, informed consent was ensured. In total, eight communities across New South Wales, Queensland, South Australia and Western Australia participated in the project. These communities represented a broad social and geographical cross-section: from cities (Sydney, Brisbane, Adelaide, Perth), regional areas (Illawarra, Batemans Bay, Broome), and remote communities. Participants came from a wide variety of ages (18-60 years of age) and backgrounds: political activists, university students, stay-at-home parents, community Elders. To ensure anonymity participants were given a pseudonym. Fifty semi-structured interviews were conducted, lasting between thirty and ninety minutes each. Ten themes were covered in interviews, including: quantity and quality of social media use; expressions and concerns of culture on social media; Indigenous identities; community formation and concerns. Drawing on Nakata's (2007) Indigenous Standpoint Theory and Cultural Interface, interview transcripts were then subject to a form of narrative discourse analysis.

As social interactions are increasingly conducted on social media, there was also an opportunity to collect additional data through an online social media-driven survey created using SurveyMonkey and promoted via Facebook and Twitter, using a snowball sampling technique. The survey was anonymous and consent was tacit, indicated by the choice to complete the survey. Simple quantitative analyses were conducted using SurveyMonkey's inbuilt statistical tools. Methodological rigour was ensured through research triangulation: using a multi-method approach and ensuring all analysis was conducted by at least two researchers.

\section{Results \& Discussion}

\subsection{A Less Daunting Way to Seek Help}

Most participants in this study esteemed social media as a place of mutual support and care, suggesting it offers a platform where users could receive support and encouragement through 
interactions with friends and family. Through posting their thoughts and feelings as 'status updates', or directly reaching out to others through comments and private messages, participants could feel supported by their online network.

In this way, some participants reported that social media is a less daunting way for users to find support and seek help in regards to suicidal ideation and behaviour, as there is less embarrassment due to the removal of the face-to-face contact:

It does make it easier. Because you might be too embarrassed or not want to talk to a person physically about it, whereasyou can just go onlineand talk to someone or even look it up on the internet. it's not as confronting. If you sat down with somebody, you think they might judge you. Whereas if you did it online you can't really tell if they're judging you, so it's less confronting (Daniel, Male, 19).

Certainly, seeking help for suicidal behaviour from mainstream formal help sources can be a daunting task for Aboriginal peoples. Farrelly (2008) found that mainstream services were typically described as being culturally inappropriate, due to a lack of awareness of Aboriginal culture, history, and a discipline base that does not account for Aboriginal frames of reference for particular aspects of health and well-being. The procedures and protocols that must be followed in order to gain access to services can be intimidating to the uninitiated, unaccustomed, or those whom the service is not directly targeting. The capacity of social media to make help-seeking less daunting is thus an appealing option.

Likewise, participants described social media, and the act of writing public posts, as offering an alternative outlet for users' feelings and thoughts, circumventing some of the social barriers to requesting help. For instance, one participant suggested:

...writing is an incredibly powerful thing, and sometimes people write stuff that they wouldn't necessarily say. So I have seen people express some really stressed, distressed feelings. .. I think people with those sorts of issues can sometimes use that as a healing mechanism. What they're keeping inside, through the mode of writing, they are actually able to get out (Chantelle, Female, 36).

This suggests social media might encourage users to seek support in situations where they would not have otherwise done so. Farrelly (2008) found that due to the close-knit nature of many Aboriginal communities, it can be very difficult for Aboriginal peoples to seek help for sensitive issues such as suicide, self-harm, sexual abuse, and family violence. Accessing informal sources of help generally involves disclosing the sensitive issue to a community member - the potential ramifications of which could be overwhelming for the individual. Farrelly (2008) noted her participants stated that fears of these potential ramifications would generally compel them to avoid disclosure altogether and avoid accessing any alternative informal help sources. This avoidance can result in such issues becoming hidden within the community - the discussion of which becomes taboo. Likewise, participants in the current study expressed concerns regarding Facebook's policy of transparency, which leaves little room for anonymous activity. If engagement with services on Facebook-mental health services for example -is publicly open for that person's network to see, it might become a further barrier to seeking help.

\subsection{An Opportunity to Offer Help}

The majority of participants described Facebook as providing an opportunity to offer help to someone they believed was at risk of suicide. For example:

That's a big part of what I am there for. And for whatever reason, there are various people that see me as a person they can trust and they do [reach out for help]. And so they lean on me to do that. And so you just try to bestrong enough to do what you can to help people (Thomas, Male, 41).

While this suggests a possible positive avenue for help-giving on Facebook, some participants were ambivalent about public calls for help. They expressed scepticism towards the genuineness and seriousness of some threats of self-harm, deeming them to be 'attention- 
seeking' rather than help-seeking. For example, one participant noted the use of suicidal status posts for the purpose of gaining likes'-a function on Facebook where other users can indicate they like' your photo or written post:

But sometimes I think it is just about getting attention. Everyone just wants to be the focus of all the posts and get all the likes. It is pretty sad when you get a lot of likes if you post that you are suicidal or feeling miserable (Maree, Female, 48).

Other participants reported they restricted their offers of help to family only, and a small number expressed an unwillingness to offer help at all, either because they feared they might give the wrong advice or because they felt annoyed by what they deemed to be attentionseeking behaviour. As one participant stated: "I told them not to advertise their problems on Facebook" (Katie, Female, 24).

\subsection{Aboriginal Facebook Suicide Prevention Strategies}

But when these barriers were not present, participants described what could be collectively understood as a continuum of Facebook suicide prevention strategies that they would implement to varying extents if they were concerned that an individual was at risk of suicide. At one end of this continuum are interventions that are relatively non-intrusive and easily implemented, such as fostering a general 'positive atmosphere' in their Facebook interactions with others, or letting people know that they are present as a source of support. Interventions then progressed to using Facebook to offer support, help, information about available services, and advice to people they felt could be at risk of suicide - generally through private messaging, or 'inboxing', as opposed to public messages. This was described as a more direct means of offering help to someone, while protecting his or her privacy:

I wouldn't do it in the public domain, you know. If it was a privatemessage, something like that, like if it's Facebook, I wouldn't be doing it so that everyone else could see. I wouldn't want to humiliate them or makethem feel targeted any more than what they already were (Lara, Female, 22).

Progressing along this continuum, it is apparent that Facebook enables some participants to actively keep an eye on people' that they may be concerned are at risk of suicide, usually by monitoring their posts and checking in with them on a regular basis:

I gave them the contact details for the counsellor at the AMS [Aboriginal Medical Service] and just let them know they were not alone and I checked in on them through the next few days (Katie, Female, 22).

Participants unanimously agreed that while social media such as Facebook can provide a platform for Aboriginal suicide help-seeking and helping, it should not replace face-to-face contact, but rather could complement it. And so the progression to the opposite end of the continuum includes Aboriginal Facebook suicide prevention strategies that utilise Facebook to enable the organisation of face-to-face assistance. Participants described rallying around with other friends on Facebook who also knew the person at risk, or contacting their friends and family to try and ascertain what may be going on and what help the person may need. This could then lead to actually getting together in person and visiting the individual at their home, and/ or calling emergency or other services. As one participant explains:

I remember one young man was writing some, well, it seemed quite suicidal thoughts on that [Facebook]. So it ended up a bunch of us actually rallying together to make sure that police were sent around and went to that person's place and it was all okay (Chantelle, Female, 36).

Some participants even reported using their own contacts in the region in which the person at risk was living, which could be interstate, to rally together and go visit the person at risk:

A couple of weeks ago, a lady from, well I don't really know her, but she's family, so I'm friends with her on Facebook. And she wrote, and I thought it was an attentionseeking thing, but she wrote, because she had a warrant out for her arrest or 
something, and she wanted to top herself off, she doesn't know what to do. So I messaged one of my cousins in Darwin and just let them know what was going on...they went around and she was all right (Louise, Female, 25).

In this respect, while the close-knit nature of many Aboriginal communities can impede Aboriginal help-seeking for suicidal behaviour, it can also be utilised with the assistance of Facebook to implement effective Aboriginal suicide prevention strategies.

\subsection{Rapid Information Dissemination}

All participants agreed that Facebook and other social media has the potential to aid Aboriginal suicide prevention by means of the provision of fast access to relevant information. This notion is empirically supported by research conducted by Silenzio et al. (2009), who used 'network modelling' to track and map the flow of suicide prevention information through Lesbian/ Gay/ Bisexual (LGB) identified social media users. Like Indigenous peoples, globally young LGBQTI people are more likely to attempt suicide than general populations. Silenzio et al. (2009) ultimately demonstrated the potential of social media in acting as a suicideprevention platform through information distribution.

Qualitative data from the current study further substantiates the results of Silenzio et al. (2009) in an Indigenous context. As many Aboriginal people are active on Facebook and other social media, it has the capacity to quickly and easily distribute information to a wideaudience. Although participants expressed some ambivalence as to whether this information would effectively reach the person at risk, it was suggested it might help friends and family within the network in identifying risk factors, taking action, and supporting them emotionally. For example:

I suppose it's good because, with younger people, I suppose their first thought would be to go on Facebook, rather than Googling depression, something like that (Lara, Female, 22).

Those sorts of things can be disseminated through social media, and really, really quickly (J acob, Male, 34).

Certainly, information for users helping to identify suicide warning signs would be beneficial given the ambivalence of many participants about whether some behaviour was attentionseeking as opposed to help-seeking. It was also suggested that campaigns through social media could help reduce the stigma around mental health and instigate much-needed discussions around the issue:

I think to start the discussion it's good. Because when [the actor] Robin Williams passed away, a lot of people were talking about it. And a lot of the statistics around suicideactually came to life, which I find a lot of peoplethat I knew, well I never knew that it was the leading cause of young adult deaths. I never knew that. So for me to actually find that out, I was taken aback by that (Louise, Female, 25).

\subsection{Mediating Tragedy - Bullying, Racism, Negativity, and Suicide Contagion}

Silenzio et al.'s (2009:2) optimistic conclusion about the efficacy of social media in reducing suicidal behaviour and attitudes came with a notable qualification, however: "Online networks have the dual potential to serve as platforms for prevention interventions as well as to serve as conduits for toxic influences such as suicide contagion." As the current study also found, the flip side of this suicide prevention capacity of Facebook and other social media is the potential for bullying, racism, negativity and suicide contagion. Some participants reported that interacting on Facebook is often a source of negative feelings about the self:

Facebook is a big problem for young people. Some people see their message, some people talking about you on Facebook, and they pass the message and pass the message. And that person, 'Oh, I've had enough, nobody like me on Facebook, might kill myself' (Sam, Female, 25). 
But at the same time, sometimes [Facebook] is the cause. Because people on there, you know, you see the bullying sides of things, that could be pushing them to, you know, feel worthless (Nicole, Female, 20).

And more worryingly:

\section{You could drive someone to suicide on Twitter (Thomas, Male, 41).}

An episode of Living Black (a television show featuring stories of interest to Australian Indigenous people) titled 'Cyber Wars' (19 April 2010) highlighted concerns about how racism online is affecting Indigenous people and communities. Furthermore, the J oint Committee on Cyber-Safety in 2013 led an 'Inquiry into Issues Surrounding Cyber-safety for Indigenous Australians' after evidence revealed that Indigenous people were increasingly subject to cyber bullying and online racism. The report confirmed that online racism does have a negative effect on Indigenous people (2013:20). It also raised concerns about the link between cyber-bullying, racism and suicide (2013:23).

Cyber bullying among Aboriginal people is a worrying trend. In 2012, Aboriginal people living in a remote location were calling for a ban on Facebook and social media, as it was believed that it was fuelling community tensions and what was referred to as "old family rivalry and blood feuds" (Michael 2012). It was reported that a street brawl took place after exchanges on social media sites reignited a long-standing dispute. While some community members were calling for a social media ban, others were against censorship and were calling for more education so the community could have a better understanding of the capacity of social media.

The other concern participants voiced about Facebook and suicidal behaviour was in regards to suicide clusters. Aboriginal suicide appears to occur in clusters at certain points in time in particular communities, and certainly more frequently than in the general population (Hunter et al. 2001). Such clusters can be described as suicide contagion, whereby exposure to suicidal behaviour appears to trigger further 'copycat' suicidal behaviour. The clearly defined, contained, shared physical environment of many Aboriginal communities results in high visibility of and exposure to suicidal behaviour. Suicides, particularly the common method of hanging, are frequently witnessed by many members of an Aboriginal community, who experience firsthand the impact such deaths have on the community. These deaths often spark a cluster of suicides in Aboriginal communities involving similar methods, gender and age groups. As Hunter et al. (2001) notes, this suggests suicide can play a catalytic role in communities through observational learning, imitation, and modelling.

One participant, who works in the health and community sector, suggested that in the aftermath of the suicide of a young Aboriginal girl, several Elders became concerned about the information being communicated via social media and the impact this may have on other young people in the community. They feared that this may foster suicide contagion. To quote at length:

We had, unfortunately, a young girl in this community commit suicideon Boxing Day just passed. And...the content went throughout the network very, very quickly. So it was a matter of, we got rung up by a couple of Aunties to get around to some of the young fellas to try to make sure everything was all right, because there was a lot of knowledge going out and a lot of hurt going around. So, look there's two folds to it. Threats and stuff are themain, and there's you know there's suicide that does happen, and how do people process that information? And when you're accessing that you've got a 1000 people in your support network but no one physically around you, I think is a different situation. I think that's what we're finding with a lot of the suicide stuff that we get. We get big clusters. That wemight have oneyoung guy do it, and then we might have three or four within that immediate social group go down the same path. And it's sort of hard to predict and manage (Dave, Male, 33).

This concern is given empirical support by Kramer et al.'s (2014) recent and controversial study on "massive-scale emotional contagion" on Facebook. Although it did not measure suicide contagion specifically, it showed negative emotion can travel through online networks. 
With an almost unrivalled sample size $(n=689,003)$, researchers altered the Facebook newsfeed algorithm in order to restrict what types of content with which individual users were presented. Through identifying and then either eliminating or emphasising content containing key emotive words and terms, the two test groups were presented with Facebook content that was either primarily negative or primarily positive (while the control group saw an unaltered 'news feed'). The experiment ran for a single week in 2012 and turned up small, but statistically significant results: those in the 'negative' group we more likely to likewise generate 'negative' content, while the 'positive' group were more likely to generate 'positive' content. Although the effects were small, "given the massive scale of social networks such as Facebook, even small effects can have large aggregated consequences" (Kramer et al. 2014:8790). It seems fear of suicidal contagion passing through online Aboriginal networks is justified.

\section{Conclusion}

This paper offers the first study of Aboriginal people's use of social media for help-seeking and help-giving not restricted to young people. It shows that Aboriginal people already consider social media such as Facebook as a place of mutual support and care. Previous research has identified significant barriers for Aboriginal people in accessing help for mental well-being, suggesting they are more likely to engage in informal methods of help-seeking. Thus the above results demonstrate Facebook offers a further, potentially vital avenue for Aboriginal people to seek help outside formal services.

Participants in this study reported being more often on the receiving end of requests for help, articulating a number of already existing help-giving practices taking place on Facebook. In mobilising help, they drew on a continuum of practices ranging from soft, informal help-giving techniques (such as creating a positive online environment) to direct intervention (in some cases involving emergency services).

But there was also some ambivalence in Facebook's efficacy in acting as a platform for helpseeking and help-giving. While overcoming several barriers to help (such as bureaucracy and cultural inappropriateness), the use of Facebook for these ends presented their own barriers. Participants were often sceptical of public calls for help, instead identifying these posts as attention-seeking. Others lacked confidence in providing help to those in need. Most concerning, however, is Facebook's potential to contribute to mental ill-health, through facilitating racial abuse and suicide contagion. Thus any potential health benefits of social media need to be weighed against these challenges.

With this in mind, we here suggest that a promising option in addressing Aboriginal suicide is to engage with these already existing - and from the accounts above, effective-practices. From this initial knowledge base, we encourage the development and evaluation of culturally appropriate suicide intervention programs, using Facebook as a platform for facilitating help. As the participants indicated, this could not replace face-to-face interventions, but would rather complement and enhance them.

The need is urgent. Aboriginal suicide is among the highest in the world (Ting and Hunter 2015). Aboriginal children under 14 years are almost eight times more likely to commit suicide than their non-Aboriginal compatriots. Aboriginal people are already engaging in help-seeking and help-giving on Facebook. Leveraging these in a thoughtful way could help reverse these tragic trends.

\section{Acknowledgments}

The authors wish to acknowledge and thank the Aboriginal people who participated in this study. Your contributions, experiences and anecdotes have enriched this area of research. Additionally, we would also like to thank the research assistants in each community who generously provided us with information on local cultural protocols. 


\section{Ethics}

This research has ethics approval from the University of Wollongong Human Research Ethics Committee. Approval granted on 26 September 2013 - 2014 and extended to 25 August 2015. Reference Number: HE13/325

\section{Funding}

This research is supported by the Australian Research Council Discovery (Indigenous), Project ID: IN130100036.References

\section{References}

Australian Institute of Aboriginal and Torres Strait Islander Studies Conference, 2010, 'Information Technologies and Indigenous Communities' http:// www.aiatsis.gov.au/research/ symposia/ Digi10/ theme.html

Callinan, T. 2014, Remote Indigenous Australians rely on Facebook to stay in touch' 29 September 2014, http:// www.sbs.com.au/news/article/ 2014/ 08/26/ remoteindigenous-australians-rely-facebook-stay-touch

Carlson, B. 2013, 'The 'new frontier': Emergent Indigenous identities and social media' in M. Harris, M. Nakata, \& B. Carlson (eds.), The Politics of Identity: Emerging Indigeneity, University of Technology Sydney E-Press, Sydney, pp. 125-146.

Carlson, B. 2014, 'Well connected Indigenous kids keen to tap new ways to save lives' The Conversation, 20 September 2014, https://theconversation.com/ well-connectedindigenous-kids-keen-to-tap-new-ways-to-save-lives-30964

Carlson, B. \& Frazer, R. 2015, “It’s like going to a cemetery and lighting a candle”: Aboriginal peoples, Sorry Business and social media', AlterNative: An International J ournal of Indigenous Peoples, 11(3).

Dudgeon, P. \& Kelly, K. 2014, Contextual factors for research on psychological therapies for Aboriginal Australians, Australian Psychologist, 49(1), pp. 8-13.

Edmonds, F., Rachinger, C., Waycott, J. Morrissey, P., Kelada, O. \& Nordlinger, R. 2012, 'Keeping intouchable: A community report on the use of mobile phones and social networking by young Aboriginal people in Victoria', Institute for a Broadband-Enabled Society, University of Melbourne.

Emery, K. 2014, 'Social Media driving racism', The West Australia, 30 June, https:// au.news.yahoo.com/thewest/wa/a/21068988/ social-media-driving-racism/

Elliott-Farrelly, T. 2004, 'Australian Aboriginal suicide: The need for an Aboriginal suicidology?' Australian e-J ournal for the Advancement of Mental Health, 3(3), pp. 1-8

Farrelly, T. 2008, The Aboriginal suicide \& self-harm help-seeking quandary', Aboriginal \& Islander Health Worker J ournal 32 (1), pp. 11-15.

Georgatos, G. 2014, 'It is racism killing our people- suicides born of racism', The Stringer, 30 J une, http://thestringer.com.au/it-is-racism-killing-our-people-suicides-born-ofracism-9152

Griffiths, K., Farrer, L., \& Christensen, H. 2010, The efficacy of internet interventions for depression and anxiety disorders: A review of randomised controlled trials', Medical J ournal of Australia, 192, S4-S11.

Gould, M., Munfakh, J ., Lubell, K., Kleinman, M. \& Parker, S. 2002, 'Seeking help from the internet during adolescence', Journal of the American Academy of Child and Adolescence Psychiatry, 41, pp. 1182-1189.

Gourash, N. 1978, Help Seeking: A review of the literature', Journal of Community Psychology, 6(5), pp. 413-423. 
Hunter, E., Reser, J., Baird, M. \& Reser, P. 2001, An analysis of suicide in Indigenous communities of North Queensland: The historical, cultural and symbolic landscape, Commonwealth Department of Health and Aged Care: Canberra. .

J oint Committee on Cyber-Safety, 2013, Issues surrounding cyber-safety for Indigenous Australians. The Parliament of the Commonwealth of Australia, 20 September 2014, http://www.aph.gov.au/parliamentary_business/committees/house_of_representativ es_committees?url=jscc/indigenous_australians/report.htm

Kral, I. 2001, 'Youth media as cultural practice: Remote Indigenous youth speaking out loud', Australian Aboriginal Studies, 1, pp. 4-16.

Kramer, A. D. I., Guillory, J . E. \&Hancock, J . T. 2014, Experimental evidence of massive-scale emotional contagion through social networks, Proceedings of the National Academy of Sciences.

Living Black, 2010, 'Cyber Wars' April 19th , Special Broadcasting Service.

Lumby, B. \& Farrelly, T. 2008, 'Aboriginal ageing and disability issues in South West and Inner West Sydney', Aboriginal \&Islander Health Worker J ournal, 32 (5), pp. 27-34.

Lumby, B. \& Farrelly, T. 2009, 'Family violence, help-seeking \& the close-knit Aboriginal community: Lessons for mainstream service provision', Australian Domestic \& Family Violence Clearinghouse. Issues paper 19, September, pp.1-24.

Luxton, D. D., J une, J . D. \& Fairall, J. M. 2012, 'Social media and suicide: a public health perspective', American J ournal of Public Health, 102, pp. 195-200.

Martin, S. 2014, Facebook used by remote teens to post suicide threats, 29 September 2014, http:/ / www.theaustralian.com.au/ national-affairs/indigenous/ facebook-used-byremote-teens-to-post-suicide-threats/ story-fn9hm1pm- 1226832109265

Michael, P. 2012, 'Indigenous leaders want to ban or censor social media including Facebook', The Courier Mail, 27 September 2014, http:// www.couriermail.com.au/ news/ queensland/ indigenous-leaders-want-to-banor-censor-social-media-including-facebook/ story-e6freoof-1226237942587

Molyneaux, H., O’Donnell, S., Kakekaspan, C., Walmark, B., Budka, P., \& Gibson, K. 2014, Social media in remote First Nation communities. Canadian J ournal of Communication, 39, pp.275-288.

Moreton-Robinson, A. \&Walter, M. 2009, Indigenous methodologies in social research. Social research methods, pp. 1-18.

Price, M. \& Dalgleish, J. 2013, 'Help-Seeking among Indigenous Australian adolescents: exploring attitudes, behaviours and barriers', Youth Studies Australia, 32(1), pp.10-18.

Manago, A. M., Graham, M. B., Greenfield, P. M. \& Salimkhan, G. 2008, 'Self-presentation and gender on Myspace', J ournal of applied development psychology, vol. 29, pp. 446-458.

Nakata, M. 2007, Disciplining the Savages: Savaging the Disciplines, Aboriginal Studies Press: Canberra.

Nakata, M. 2002, Indigenous knowledge and the Cultural Interface: Underlying issues at the intersection of knowledge and information systems, IFLA J ournal, 28(5/ 6), pp. 281-291.

O'Carroll, A. 2013, 'An analysis of how rangatahi Maori are using social networking sites', MAI J ournal, 2(1), pp. 46-59.

Pascoe, B. 2014, Dark Emu, Magabala Books: Broome.

Paddock, C. 2012, 'Facebook use feeds anxiety and inadequacy says small study', Medical News Today, 30 J une, http:/ / www.medicalnewstoday.com/ articles/ 247616.php 
Pink, B. \&Allbon, P. 2008, The health and welfare of Australia's Aboriginal and Torres Strait Islander peoples, 2008. Catalogue no. 4704.0, Australian Bureau of Statistics: Canberra.

Radoll, P. 2012, Information and communication technologies in the classroom: Implications and considerations', Aboriginal and Torres Strait Islander Education: An Introduction for the Teaching Profession, Cambridge University Press: Port Melbourne, pp. 113-130.

Ranzijn, R., McConnochie, K. R. \&Nolan, W. 2009, Psychology and Indigenous Australians: Foundations of cultural Competence, Palgrave Macmillan: Australia.

Rauch, S. \& Schanz, K. 2013, 'Advancing racism with Facebook: Frequently and purpose of Facebook use and the acceptance of prejudiced and egalitarian messages', Computers in Human Behaviour, 29(3), pp. 610-615.

Reser, J . P. 1991, 'Aboriginal mental health: conflicting cultural perspectives' in J . Reid and P. Trompf (eds.), The health of Aboriginal Australia. Harcourt Brace \& Company: Marrickville, Australia.

Rigney, L. 1997, Internationalization of an Indigenous anticolonial cultural critique of research methodologies: A guide to Indigenist research methodology and its principles', Wicazo Sa Review, pp.109-121.

Rigney, L. 2011, 'Indigenous education and tomorrow's classroom: Three questions, three answers', N., Milgate, G., Bell, HR (ed/s.), ACER Press, pp. 35-47.

Rickwood, D., Deane, F., \&Wilson, C. 2007, When and how do young peopleseek professional help for mental health problems?', Medical J ournal of Australia, 187(7), pp. 35-39.

Robinson, J., Rodrigues, M., Fisher, S., \& Herman, H. 2012, 'Suicide and Social Media: Findings from the Literature Review, Young and Well Cooperative Research Centre, Melbourne.

Ruder, T. D., Hatch, G. M., Ampanozi, G., Thali, M. J. \& Fischer, N 2011, 'Suicide announcement on Facebook', Crisis, 32, pp. 280-2.

Rushing, S.C. \& Stephens, D. 2011, Use of media technologies by Native American teens and young adults in the pacific northwest: Exploring their utility for designing culturally appropriate technology-based health interventions', The Journal of Primary Prevention, 31(1-2).

Silenzio, V. M., Duberstein, P. R., Tang, W., Lu, N., Tu, X. \&Homan, C. M. 2009, 'Connecting the invisible dots: reaching lesbian, gay, and bisexual adolescents and young adults at risk for suicide through online social networks' Social Science \& Medicine, 69, pp. 46974.

Tatz, C. 2001, Aboriginal suicideis different: A portrait of life and self-destruction, Aboriginal Studies Press: Canberra.

The Elders' Report into Preventing Indigenous Self Harm \& Youth Suicide, 2014, Culture is Life Campaign, 26 September 2014, http://apo.org.au/research/elders-reportpreventing-indigenous-self-harm-and-youth-suicide

Ting, I. \& Hunter, F. 2015, 'Indigenous suicide rate 12th highest in the world', The Sydney Morning Herald, 13 J uly, http://www.smh.com.au/national/health/indigenoussuicide-rate-12th-highest-in-the-world-20150710-gi9jyn.html

Vitak, J. 2014, 'Facebook makes the heart grow fonder: relationship maintenance strategies among geographically dispersed and communication-restricted connections'. In Proceedings of the 17th ACM conference on Computer supported cooperative work \& social computing (CSCW '14). ACM, New York, NY, USA, 842-853.

Westerman, T. 2010, 'Engaging Australian Aboriginal youth in mental health services', Australian Psychologist, 45(3), pp. 212-222. 
Whiteman, H. 2013, Could Facebook be making you miserable?', Medical News Today, 30 J une, http:// www.medicalnewstoday.com/articles/ 264902.php

Worrall, A. 2015, Facebook introduces suicide prevention tools', The Sydney Morning Herald, 30 J une,_http:// www.smh.com.au/national/facebook-introduces-suicide-preventiontools-20150227-13qciq.html

Zuberick, S., Silburn, S., Lawrence, D., Mitrou, F., Dalby, R., Blair, E., Griffing, J ., Milroy, H., De Maio, J., Cox, A. \& Li, L. 2005, The social and emotional wellbeing of Aboriginal children and young people, The Western Australian Aboriginal Child Health Survey: The Social and Emotional Wellbeing of Aboriginal Children and Young People, Curtin University and Telethon Institute for Child Health research, Perth.

Copyright: (c) 2015 Carlson, Farrelly, Frazer \& Borthwick. This is an open-access article distributed under the terms of the Creative Commons Attribution-NonCommercial 3.0 Australia License, which permits non-commercial use, distribution, and reproduction in any medium, provided the original author and AJ IS are credited.

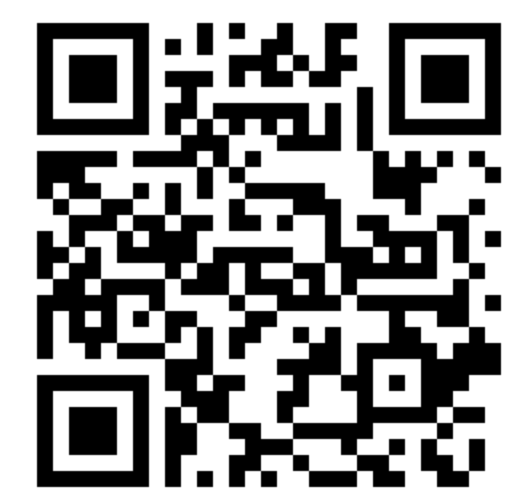

